Originalveröffentlichung in: Zeitschrift für Assyriologie und vorderasiatische Archäologie 94,

2004, S. $226-249$

\title{
Wörter und Sprichwörter: der zweisprachige Schultext HS 1461
}

\author{
von Manfred Krebernik - Jena
}

Die im folgenden publizierte altbabylonische Tafel HS 1461 der Jenaer HilprechtSammlung enthält einen zweisprachigen Text ungewöhnlicher Struktur: auf einen lexikalischen Abschnitt in Listenform folgen zwei Sprichwörter, deren zweites durch zwei Lemmata mit dem lexikalischen Abschnitt verknüpft ist. Darüberhinaus bietet der Text zahlreiche bislang nicht belegte Ausdrücke, Schreibungen und Gleichungen.

Die Tafel HS 1461 (Höhe ca. $10 \mathrm{~cm}$; Breite 5,8 cm; maximale Dicke ca. 2,3 cm) ist auf der Vorderseite und auf dem oberen Teil der Rückseite relativ eng beschrieben (Zeichenhöhe 3 bis $4 \mathrm{~mm}$ ). Aus paläographischen, orthographischen und sprachlichen Gründen kann die Tafel in die altbabylonische Zeit datiert werden. Die Schachtel, in der sie aufbewahrt wird, ist von Hilprechts Hand mit der Signatur „Ni-141 IV“ beschriftet. Mit einiger Wahrscheinlichkeit stammt HS 1461 somit aus Nippur - was durch textinhärente Merkmale gestützt wird (vgl. i 9; ii 11-14 mit Kommentar) - und wurde dort während der IV. Grabungskampagne der „Babylonian Expedition" gefunden.

Die Vorderseite umfaßt zwei Kolumnen zu je 26 Zeilen, die jeweils ein sum. Lemma mit akk. Entsprechung enthalten. Dieses Schema setzt sich auf der beschädigten Rückseite zunächst fort (Kolumnen iii und iv mit je 3 Zeilen). Es folgen, äußerlich nicht abgesetzt, acht über die ganze Tafelbreite laufende Zeilen (Kolumne v), die zwei sum. Sprichwörter mit jeweils folgender akk. Übersetzung enthalten. Das zweite ist durch die Lexeme ti-ti und te-te-h hé mit dem lexikalischen Abschnitt verknüpft (ii 6-7). Das Textende ist durch eine Doppellinie markiert. Der leere Rest der Rs. trägt Tilgungsspuren sowie zwei schwache, vertikal bzw. horizontal verlaufende, sich rechtwinklig schneidende Doppellinien. Die linke untere Tafelecke ist beschädigt, auf dem unteren (von der Vs. aus oberen) Tafelrand ist als Rest eines Subskripts der Name der Schreibergöttin Nisaba erhalten.

Unter den sum. Lemmata finden sich viele hapax legomena, bei denen es sich wohl größtenteils um unorthographische Schreibungen handelt. Das Akkadische weist durchwegs korrekte, meist mit Mimation versehene Kasusendungen auf. Das akk. Syllabar entspricht im wesentlichen altbabylonischen Gepflogenheiten, umfaßt jedoch auch die ungewöhn- 


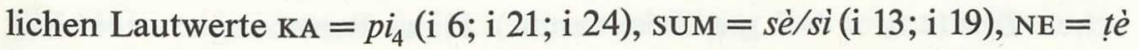
(i 9) und NI $=i(\mathrm{v} 4)$, die bis auf den ersten im Sumerischen beheimatet sind. Charakteristisch ist ferner die konsequente Darstellung von auslautendem -/û/ durch $\mathrm{Cu}$-ù (gegenüber gebräuchlicherem $\mathrm{Cu}$-ú).

Der lexikalische Abschnitt gehört keiner bekannten Serie an. Die Lemmata folgen keinem durchgängigen Thema oder Gliederungsprinzip, doch lassen sich einzelne, durch formale und/oder semantische Gemeinsamkeiten konstituierte Gruppen erkennen. Die längste bildet den Anfang der Liste und umfaßt 16 mit bàd gebildete Ausdrücke (i 1-16). In der Folge finden sich nur noch Zweier- und Dreiergruppen:

i 17-18: Ausdrücke mit ga1-ga1

i 19-20: GALAM $^{1}$

i 22-23: Ausdrücke mit s a $\hat{g}$

i 25-26: Begriffsfeld "Wolke“

ii 4-5: Begriffsfeld "Opfer" (?)

ii 6-7: Begriffsfeld „belästigen“ (ähnlichlautende sum. Wörter)

ii 8-10: Begriffsfeld „Essen und Trinken“2

ii 11-14: Begriffsfeld „Schaf" bzw. „Fleisch"3

ii 16-17: Ausdrücke mit še-er, Begriffsfeld „Glanz“

ii 25-26: Ausdrücke mit si, Begriffsfeld „Horn“

iii 1-2: Begriffsfeld „waschen“ (?)

Die erste Gruppe ist mit Proto-Izi 318-333 verwandt. Auch typologisch ist unsere Liste mit Izi und ähnlichen "akrographischen“ Listen vergleichbar, wie sie in MSL 13 beschrieben (s. dort S. 3 f.) und ediert sind. Eine Gemeinsamkeit von HS 1461 und KÁ.GAL (MSL 13, 61-88; 227-261) besteht darin, daß beide Listen mit Bautermini beginnen.

Von besonderem Interesse ist die in HS 1461 sich manifestierende Intertextualität: einerseits werden auf der Tafel „lexikalisches“ und „literarisches" Genre über die Lemmata ti-ti und te-te-hné, die sowohl in der lexikalischen Liste wie auch in dem abschließenden Sprichwort erscheinen, miteinander verknüpft; andererseits bilden dieselben Lemmata eine Schnittstelle mit den grammatischen Texten der altbabyloni-

1 Die Zeilen 17-21 sind darüberhinaus durch den gemeinsamen Lautkomplex /gal/ bzw. /g al/ miteinander verknüpft.

2 Man könnte auch die Zeilen ii 5 und ii 8-10 unter dem Oberbegriff „Essen und Trinken " zusammenfassen, in welchem Falle ii 6-7 einen (fehlplazierten) Einschub darstellen würden.

${ }^{3}$ Schließt wohl inhaltlich an die vorige Gruppe an. Zur Verwandtschaft mit dem Nippur-Vorläufer von Hh XIII s.u. Kommentar. 
schen Zeit (s.u. ii 6-7 mit Kommentar). Diese Beziehungen verweisen ebenso wie die im Subskript genannte Schreibergöttin Nisaba auf die „Schule“, d.h. die Schreiberausbildung, als Entstehungshintergrund von HS 1461.

Beziehungen zwischen lexikalischen und literarischen Texten sind seit frühdynastischer Zeit zu beobachten ${ }^{4}$. Im altbabylonischen Nippur bildeten sum. Modellverträge und Sprichwörter die Fortsetzung des Listenstudiums und den Übergang zum Studium literarischer Texte ${ }^{5}$. Kombinationen von mathematischen oder lexikalischen Übungen mit einem Sprichwort kommen typischerweise auf runden Tafeln vor, selten dagegen auf mehrkolumnigen Tafeln, wie sie auf fortgeschrittenerem Niveau verwendet wurden ${ }^{6}$. Altbabylonische Beispiele für die Kombination von lexikalischen Übungen mit Sprichwörtern bieten die fragmentarischen Tafeln N $5213^{7}$ und N 61198, deren Typ nicht sicher erkenntlich ist ${ }^{9}$. Ähnliche Fälle sind auch im I. Jt. bezeugt: so enthalten die beiden Übungstafeln BM $60187++{ }^{10}$ und BM $64483++{ }^{11}$ einsprachige lexikalische Exzerpte und akk. Sprichwörter (in dieser Reihenfolge).

Die in HS 1461 greifbaren Beziehungen zwischen lexikalischen Listen, Sprichwörtern und grammatischen Texten zeugen von der Komplexität der altbabylonischen Schreiberausbildung und lassen Schlüsse auf das zugrundeliegende Curriculum zu, die Bekanntes zu bestätigen und zu ergänzen scheinen. Inhaltlich und vor allem auch typologisch ist HS 1461 allerdings, soweit ich sehe, bisher singulär, die Interpretation daher mit manchen Unsicherheiten behaftet. Für eine genauere Einordnung von

${ }^{4}$ Zur Intertextualität im allgemeinen sowie zur Verwendung von Sprichwörtern in Briefen und literarischem Kontext s. Hallo (1990). Zu Beziehungen zwischen lexikalischen und literarischen Texten in frühdynastischer Zeit s. Krecher (1992, 293 f.) und Krebernik (1997, 190f.). Für die altbab. Zeit s. Civil (1987), Michalowski (1998) und insbesondere Veldhuis $(1997,126 \mathrm{f}$.).

5 Verträge und Sprichwörter bilden nach N. Veldhuis den 4. Abschnitt des Curriculums, vgl. die Übersicht Veldhuis $(1997,63)$. Zu korrespondierenden Tafelfunden in Haus F s. Robson (2001).

6 Vg1. Alster (1997, xxi).

7 Die Vs. enthält Sprichwörter, die Rs. ein Exzerpt aus Proto-Izi, s. Alster (1997, 231; 235; P1. 70) bzw. MSL 13, S. 14 Text A1.

8 Alster (1997, 292; P1.102). Die Vs. enthält Sprichwörter, die Rs. ein Exzerpt aus dem Nippur-Vorläufer von Hh XIII (entspricht MSL 8/1, S. 84-85, Z. 69?-115).

9 Einsprachig; Sprichwörter auf der Vorderseite, lexikalischer Teil auf der Rückseite; wahrscheinlich „Type II“ zuzurechnen. Zu den verschiedenen Typen altbabylonischer Schultexte s. Veldhuis (1997, 28-39) mit Rückweis auf M. Civil, MSL 13, $27 \mathrm{f}$.

10 Gesche (2001, 460-463).

11 Gesche (2001, 477-482). 
HS 1461 in den Kontext der Schreiberausbildung sind folgende Beobachtungen und Überlegungen relevant:

(1) Die Handschrift ist zwar geübt, klein und stellenweise sehr eng, doch legen etliche Tilgungen bzw. Korrekturen sowie aberrante Zeichenformen ${ }^{12}$ den Schluß nahe, daß die Tafel nicht von einem Lehrer, sondern von einem fortgeschrittenen Schüler beschrieben wurde. Wegen des mangelnden Vergleichsmaterials muß allerdings offen bleiben, ob der Text gemäß einer bestimmten Aufgabenstellung vom Schüler selbst kompiliert, oder ob er nach Diktat oder nach Vorlage geschrieben wurde.

(2) Auf logographisch-syllabographischer Ebene fallen zahlreiche Idiosynkrasien ins Auge. Sie sind möglicherweise nicht als bloße „Rechtschreibfehler" zu werten, wie sie besonders beim Schreiben nach Diktat entstehen können, sondern entsprechen vielleicht dem didaktischen Zweck der Schreibübung, nämlich mit den aktuellen, noch unvollkommenen Zeichenkenntnissen auszukommen oder gar zu „experimentieren“.

(3) Der lexikalische Abschnitt zeigt Anklänge an akrographische und thematische Listen, wie sie im mittleren Ausbildungsstadium ${ }^{13}$ studiert wurden.

(4) Es kommen zwar zusammengesetzte Zeichen wie Lú!? $\times^{r} \mathrm{X}^{7}$, $\mathrm{KA} \times \mathrm{NE}$ (ii $23 \mathrm{f}$.) ${ }^{14}$, aber anscheinend keine aus mehreren selbständigen Zeichen bestehende Schreibungen vor, wie sie vorwiegend in der Liste „Diri“ zusammengestellt waren; solche Zeichengruppen wären demnach einem fortgeschritteneren Stadium des Curriculums vorbehalten gewesen.

(5) Die Kombination von lexikalisch-orthographischer Übung mit Sprichwörtern ergibt einen terminus ad quem für die Einbeziehung letzterer ins Curriculum.

(6) Die Tatsache, daß die im lexikalischen Teil sowie in einem der Sprichwörter vorkommenden Lemmata ti-ti und te-te-hé auch in den Paradigmen der grammatischen Texte (OBGT) benutzt wurden, weist darauf hin, daß auf der hier dokumentierten Curriculumsstufe auch bereits grammatische Texte studiert wurden. Dieser Schluß wird durch die zweisprachig dargebotenen Sprichwörter gestützt.

\footnotetext{
12 So ist $\mathrm{udu}$ ! in ii $13 \mathrm{f}$. nur anhand von Parallelen zu identifizieren. Weitere unklare Zeichen finden sich in i 12,16 ; ii $1,15,23,24$; iv 2,3 ; v 1 .

13 Zweiter und dritter Abschnitt des Curriculums nach Veldhuis (1997, 63).

14 Beide Kompositzeichen kommen in Proto-Ea vor: Z. 625-631 (Komposita von LÚ) bzw. Z. 311-334 (Komposita von KA).
} 


\section{HS 1461}

Vs.

i

i

i

i

i

i$$
\text { (1) }
$$

i

i

i

$$
\text { i }
$$$$
\text { i }
$$

$$
\text { i }
$$

$$
\text { i }
$$

$$
\text { i }
$$

i$$
\text { i }
$$

$$
\text { i }
$$




\begin{tabular}{|c|c|c|}
\hline 13 & udu!-si-si-ga & $k i-h u-d u-u m$ \\
\hline 14 & udu ${ }^{!}-\left\{\mathrm{sI}^{! ?}\right\}-\mathrm{ri}^{\urcorner}-\mathrm{ri}-\mathrm{a}$ & pu-ru-su-um \\
\hline 15 & úugu-GÁL? & $k a-s i-B u$ \\
\hline 16 & še-er-gán & ba-ri-ru-um \\
\hline 17 & še-er-zi & ša-ru-ru-um \\
\hline 18 & 1ú-ḩi-a & gi-it-ma-lu-tum \\
\hline 19 & ŠUBUR.UR-bala & na-ba-al-ku-tum \\
\hline 20 & zú-ar & $k u-z a-z u-u m$ \\
\hline 21 & $e-1 a m-m a$ & $m e-k u-\dot{u}$ \\
\hline 22 & UD & di-qá-ru \\
\hline 23 & LÚ!? $\times^{r} X^{\urcorner} \cdot R I$ & ha-na-qum \\
\hline 24 & $\mathrm{KA} \times \mathrm{NE}-\mathrm{ZAR} ? \cdot \mathrm{ZAR} ?$ & $e-z e-z u$ \\
\hline 25 & si-gi-ri & $q a^{-}-a r-n u$ \\
\hline 26 & si-GI & 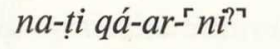 \\
\hline & & \\
\hline 1 & {$[x] \cdot{ }^{r} x \cdot x \cdot x^{\urcorner}$} & ' $m e-s u '-u m$ \\
\hline 2 & {$[x . x]-g u-1 a$} & ma-aš-ha-tum \\
\hline 3 & 'níg ${ }^{\urcorner}-s a-s a$ & $q a ́-a l-q a ́-l u$ \\
\hline 1 & {$[x-s u k]$ ud } & $z u-q \dot{u}-r[u]$ \\
\hline 2 & NI.ZU?.HAR & $E^{?}-K u^{r}{ }^{r}{ }^{\urcorner}\left[-x^{?}\right]$ \\
\hline 3 & dug?-nu-ub/uh!?-rRu & la ba-ši-i $[l]-{ }^{r} t u$ \\
\hline
\end{tabular}

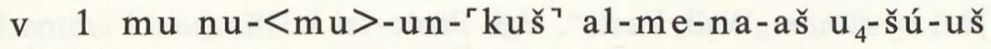
gú!?-níĝ - àr-r ra ${ }^{\urcorner}$šèg!?(A.AN!?)-gin 7

v 2 ugu-ĝá al-\{x x x $\}$-biz-biz-biz-e

v 3 aš-šum al-ma-na-ku $u_{4}$-mi-ša-am

v 4 ki-ma za- ${ }^{r} i^{?}{ }^{\urcorner}$mu-di-im e-li-ia, i-ta-na-tu-uk

v 5 hu-ru ti-ti ur-sag-e-ne

v 6 lil te-te-hé á-tuku-ne

v $7 a$ - h̆h'-ru-um mu-re-ši-mi qá-ra-di-im

v 8 li-lu-um mu-ki-id be-li e-mu-qi

(Rest der Rs. getilgt bzw. unbeschrieben)

Rd. [ $\quad]^{r} x^{\urcorner}$nisaba 


\section{Kommentar}

i 1-16 Der Abschnitt mit bàd gebildeter Lemmata weist Ähnlichkeit mit Proto-Izi I 318-333 (MSL 13, 28) auf (übereinstimmende Lemmata in Fettdruck):
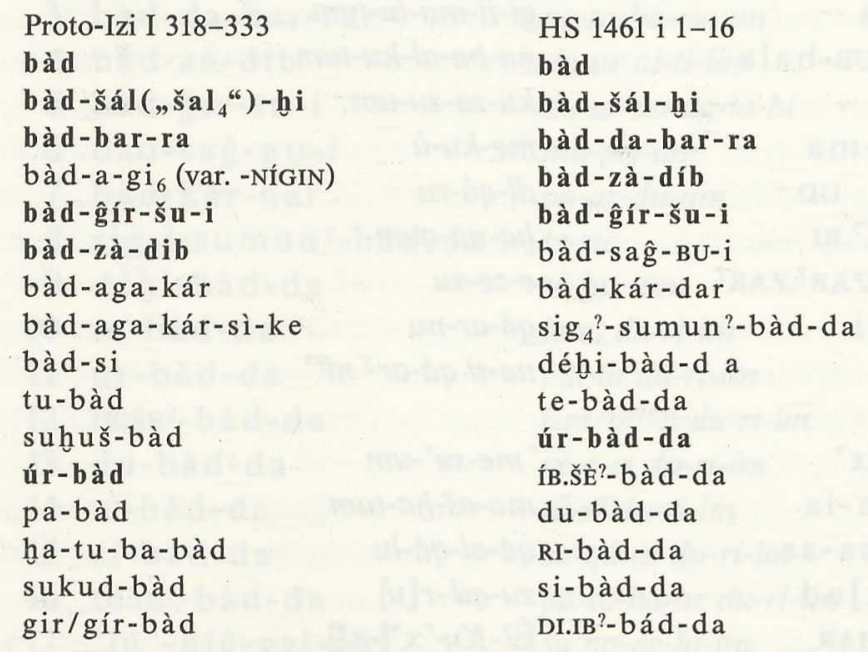

Von bàd handeln ferner Erimhuš III 7-9 (MSL 17, 47) und Nabnītu XXV 145-150 (MSL 16, 227).

i 1 [b] àd = dūrum, „Wall, Feste“. Vgl. Proto-Izi I 318.

i 2 bàd-šál-hi = šulhûm „Außenwall“. Vgl. Proto-Izi I 319. Neben der hier vorliegenden Schreibung mit sìLA = šál (in MSL 13, 28: 319 š $1_{4}$ transliteriert) ist (jünger) bàd-šu 1-ḩi gebräuchlich.

i 3 bà d-da-bar-ra = pat(t)abarrum. Vgl. Proto-Izi I 320 (bàdbar-ra). Das akk. Lemma ist offensichtlich sum. Ursprungs; es kommt auch in i 16 vor und bildet dort den Status constructus pat(t)abar, der nicht zu einem /a/-Auslaut des sum. Wortes stimmt. Das sum. Kompositum weist je eine a-haltige Endung bei bà $d$ und bar auf, die vielleicht als Fehler bzw. als bloß graphische Auslaut-Indikatoren zu werten sind. Falls die „Außenseite der Mauer" gemeint ist, würde man in Analogie zu an-šà, ir i-bar und anderen Komposita dieses Typs *bà d-bar erwarten.

i 4 bà d-zà- $\mathrm{díb}(\mathrm{KU})=d \bar{u} r$ nītim „Umfassungsmauer“. Vgl. Proto-Izi I 323 ( bàd-zà-dib).

i 5 bàd-ĝ́r-šu-i = dūr naglabi. Vgl. Proto-Izi I 322. Gemeint ist eine Partie des metaphorisch naglabu „Schermesser" genannten Körperteils. AHw. s.v. naglabum 2 deutet den Ausdruck als „Hüfte“, „Weichen“, mit CAD s.v. naglabu A dürfte aber das „Schulterblatt“ (bzw.die „Schulter") gemeint sein. Ominöse Befunde am dūr naglabi des Schafes be- 
handeln YOS 10, 47: 61-64 (du-ur na-ag-la-bi-im) und YOS 10, 48: 1 (du-ur šU.I).

i 6 bàd-sa $\mathrm{g}-\mathrm{BU}-\mathrm{i}=m u-p i_{4}(\mathrm{KA})-u m$. Das sum. Lemma und seine akk. Entsprechung sind bisher nicht belegt. Das akk. Wort ist wohl (als Substantiv der Nominalform maPRaS) zu wapûm, sichtbar sein/werden, (strahlend) erscheinen“" zu stellen. sa ĝ-BU(-BU)-i wird mit šarāru bzw. Gtn šitarruru geglichen, deren genaue Bedeutung nicht feststeht; die Wörterbücher setzen jeweils fragend an: „sich vorbeugen, sich neigen (zum Weitergehen)“ (AHw.) bzw. „to go ahead“ (CAD), doch könnten die Verben aufgrund unserer Gleichung zu šarüru "Glanz“ gehören. In Kagal B 252-255 (MSL 13, 237) finden sich ferner die Gleichungen s a $\hat{\mathrm{g}}-\mathrm{BU}=$ amāru ,sehen“, arku „lang“, ša-ku-zu (unklar).

i 7 bà d-kár-dar = pardum. Vgl. Proto-Izi I 324 (bàd-aga-kár)? Sum. kár-dar könnte eine unorthographische Schreibung für gàr-dar = sakāpu „zurückstoßen“ sein, s. dazu Römer $(1988,40)$ mit Lit. Das akk. Äquivalent ist wohl Verbaladjektiv von parādu „erschrecken“, das oft im Sinne von „schrecklich" gebraucht wird. bàd-kár-dar wäre demnach eine „abschreckende (Mauer)“.

i 8 sig $_{4}$ ?-s um un ?-bà d-d a = têru. Die Lesung des sum. Lemmas ist nicht völlig sicher, anstelle von $\mathrm{SIG}_{4}$ und BAD (= sumun) kommen (weniger wahrscheinlich) auch LUM bzw. NU in Betracht. Die akk. Entsprechung ist sicherlich mit dem in AHw. s.v. tēru V „ein Bauteil“" gebuchten Lemma identisch.

i 9 déhi (UM)-bàd-da = tehûtum. Diese Gleichung findet sich mit derselben Schreibung tè-hu-tum (und Glosse dè-hi) auch in der NippurRezension von Proto-Aa (MSL 14, 96 Z. 185:1). Aa II/5 = 20 bietet dafür te $e_{4}(\mathrm{TE})$-hu-tum (MSL 14, 344 Z. 16; mit Ausspracheglosse di-ih). Einen Kontextbeleg dieses Terminus liefert YOS 5, 181, Z. 26f.: 20 GUR Á LÚ.HUN.GÁ ša al’-ta-ra-am; ša BÀD te (DI)-hu-ú-tim i-pu-šu „20 Kor (Gerste) als Lohn für die Mietarbeiter von A., welche die ...-Mauer bauten“. Das Wort wird in AHw. s.v. dehûtu $(m)$ fragend als „Gedrücktheit“ gedeu-

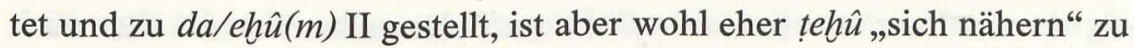
stellen. $\mathrm{Zu}$ verbalem déhyi (tehge) s. Civil (2002, 67 und 70).

i 10 te-bàd-da = lèt dūrim „Wange der Mauer“.

i 11 ú $\mathrm{r}-\mathrm{b}$ àd-da = išid dūrim „Fundament der Mauer“. Vgl. Proto-Izi I 329 (úr-bàd).

i 12 ÍB.š̀̀?-bà d-d a $=i$-ni/ir!-ti dūrim. Der sum. Ausdruck ist mir unklar. Von 1 b = qablum ausgehend würde man etwas wie „Flanke der Mauer" erwarten. Der akk. Ausdruck könnte - falls nicht $i$-ir $r^{\dagger}-t i$ „Brust" zu lesen - mit enētu „abplatten“ (AHw.) zu verbinden sein: initti als status constructus einer PiRiSt-Form *inittum? 
i $13 \mathrm{du}-\mathrm{b}$ àd-da $=s \bar{e} t$ dūrim (zu Verteidigungszwecken dienender) „Mauervorsprung“. Mit dem sum. Eintrag ist vielleicht tu - bàd in ProtoIzi $327 \mathrm{zu}$ vergleichen. Aufgrund der akk. Entsprechung wäre sum. sibà d erwarten (vgl. CAD s.v. sītu $\mathrm{A}$ ), für das i 15 jedoch die Übersetzung suqat dūrim bietet. Möglicherweise liegt eine Vertauschung von du-bàd $\mathrm{d} \mathrm{a}$ (für t tù $\mathrm{n}-\mathrm{b}$ à $\mathrm{d}-\mathrm{d} \mathrm{a}=$ suqat dūrim) und $\mathrm{s} \mathrm{i}-\mathrm{b}$ à $\mathrm{d}-\mathrm{d} \mathrm{a}=$ sīt dūrim vor.

i 14 RI-bàd-da = turri dūrim „Band der Mauer“. Anstelle von RI (W. Sallaberger [mündlich] denkt an DAL $=$ tallu „Querholz“) könnte man auch MÙš! in Erwägung ziehen. Das Regens der akk. Genitivverbindung ist wohl mit turru (< sum. dur) „Band“ zu identifizieren.

i $15 \mathrm{si}$-bàd-da = suqat dürim „Kinn der Mauer"; vgl. Kommentar zu i 13 .

i 16 DI.IB?-bàd-da = pa-ta-ba-ar dūrim „... der Mauer“. DI und mutmaßliches IB stoßen zusammen. Eine Emendation zu eššeb!(KI!.IB) = hudādu (oder huțạtu?), etwa „Lehmgrube“ (s. Sallaberger 1996, 9), wird von der akk. Entsprechung nicht nahegelegt. Diese enthält als Regens der Genitivverbindung das in i 3 mit bàd-da-bar -ra geglichene Substantiv (im Status constructus).

i 17 lú-ní ĝ-gal-gal = ša narbîm „Großer“. Dieselbe Gleichung bietet die altbabylonische Lú-Liste (A 110 [MSL 12, 161]: ša narbu'i; B iv 3 [ibd. 182]: ̌̌a narbî).

i 18 gal-gal-di = ša narbîm-ma. Akk. -ma steht hier mit Bezug auf die vorige Zeile in der Bedeutung „ebenfalls“. In Izi H 260 (MSL 13, 208) findet sich der sum. Ausdruck mit der wörtlichen Übersetzung $q \bar{a} b$ narbî „Großes sprechend“.

i 19-20 GALAM mit seinen verschiedenen Lesungen und Bedeutungen bildet die Gruppe Proto-Izi I 412-417 (MSL 13, 30f.).

i 19 galam = simmiltu „Leiter(sprosse)“, „Treppe(nstufe)“. Neben üblicherem gišs $\mathrm{kun}_{5}$ (TUR.ŠE $) / \mathrm{kun}_{4}(\mathrm{I} . \mathrm{LU})=$ simmiltu ist auch die hier vorliegende Gleichung lexikalisch bezeugt (mit Determinativ GIš): $\mathrm{Hh}$ VIIA 107 (MSL 6, 92: gišg ala m = s.); Erimhuš II 275 (MSL 17, 41: gišg al a $\mathrm{m}-\mathrm{ma}=$ MIN [i.e. simmiltu] šupali). Dasselbe Wort ist älter auch in der Schreibung gišg a-1am belegt. $\mathrm{Daß}$ gišg a-1am ein Bestandteil von

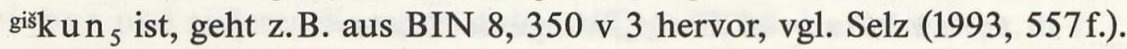

i 20 GALAM = irtum „Brust“. Diese Gleichung (anstelle von gewöhnlichem GABA = irtum) kann ich sonst nicht nachweisen.

i 21 maš-dâgal = nipi erșētim, ,(mit) Unkraut (bewachsene Fläche)“. Die Interpretation stützt sich auf den akk. Ausdruck, der gewöhnlich sum. kislah (KI.UD) oder kankal(KI.KAL) entspricht. Das sum. Lemma läßt sich partiell mit máš-a-šà-ga $=$ nipi ${ }^{\prime}$ eqli in Ana itti-šu I iv 77 (MSL 1, 14) verbinden. Dasselbe Wort könnte in Proto-Izi 339 vorliegen 
(MSL 13, 50; dort bar-dagal umschrieben). Sollte maš-daĝal mit dem gewöhnlich logographisch (ú)in-nu-uš geschriebenen Pflanzennamen maštakal identisch sein?

e-er-șe-e-tim. Die öfters anzutreffenden plene-Schreibungen sind vielleicht Indizien dafür, daß die von den anderen semitischen Sprachen abweichende feminine Form des akk. Wortes für „Erde“ auf ein analog zu šamû „Himmel“" gebildetes Pluralwort erșētum zurückgeht, das als Singular umgedeutet (und umgeformt) wurde, vgl. die analogen Fälle šî̄ātum „Wolle“ und išătum „Feuer“".

i $22 \mathrm{sa} \hat{\mathrm{g}}-\mathrm{TUM}=$ qanānum. Diese Gleichung kann ich anderswo nicht nachweisen. Von den drei in Frage kommenden akk. Verben ganānu „einschließen“, k/qanānu „einrollen“, qanānu „nisten“ ist das zweite am wahrscheinlichsten, vgl. vielleicht Nabnītu XXI 11 (MSL 16, 191): s a $\hat{g}$ íb-ši-ak-a = MIN (i.e. kanānu) ša amēli.

i 23 s a $\mathrm{g}-\mathrm{ba}-\mathrm{a}=$ mamītum "Eid“. Ähnliche Gleichungen bieten Ana itti-šu VI i 34 und VII iv 23 (MSL 1, 77 bzw. 104: s a g - ba = m.); Nabnitu IV 315 (MSL 16, 89: sa $\mathrm{g}-\mathrm{ba} / \mathrm{bi}=m$.); Erimhuš VI 81 (MSL 17, $\mathrm{sa} \hat{\mathrm{g}}-\mathrm{ba}=m$.).

i 24 dungu(IM.DIRI)-sír = šapitu "dichte (Wolke)“. Die Gleichung

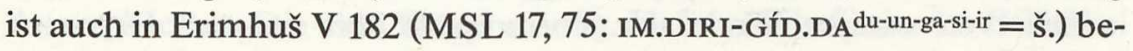
zeugt.

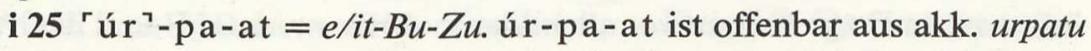
„Wolke“ entlehnt, dem gewöhnlich das (in der vorigen Zeile enthaltene) DUNGU entspricht. $e / i t-B u-Z u$ ist ein substantiviertes Verbaladjektiv des Gt-Stammes. Das zugrundeliegende Verbum (primae Aleph oder primae Nun) ist unsicher, in Frage kommen etwa epēsu „schwierig sein“ oder napașu „schlagen“, „stoßen“.

i $26[\mathrm{x}]-\mathrm{ma-at}=$ šalmu „wohlbehalten“. Im Sum. liegt wohl ein akk. Lehnwort (< šalmat) vor, zu ergänzen wäre dann [sal], [šal] oder auch [silim].

ii $11 \mathrm{i}-\mathrm{ib}-\mathrm{KA}-\mathrm{S} E \mathrm{ES} / \mathrm{AM}^{! ?}={ }^{\mathrm{r}} h u / r i^{\top}-[\mathrm{x}]-{ }^{r} \mathrm{x}^{\top}$. Unklar; die Grenze zwischen sum. und akk. Lemma ist unsicher, ŠEŠ/AM!? könnte auch zu letzterem gehören.

ii 2 bar-tab $=a-\check{s} u-\grave{u}$. Diese Gleichung könnte mit Proto-Izi 339 (MSL 13, 50: b a r-ta $\mathrm{b}^{a-k u-u}$ ) zu verbinden sein, wobei KU oder šU (das in unserem Text sehr deutlich ist) fehlerhaft wäre. PSD 2, 130, setzt fragend ein bar-tab „cripple“ an, kann aber nur auf den zitierten Beleg und das dort folgende $\mathrm{bar}-{ }^{r} \mathrm{tab} ?{ }^{?}$ ma-ku-ú $\mathrm{rxx}^{\urcorner}$(Proto-Izi 340) verweisen.

ii 3 sig = in?-bu oder še?-er-pu. Die Gleichung sig = inbu „Frucht“" ist mir nicht nachvollziehbar. Sollte šerpu zu lesen sein, so läge ein Nomen der Form PiRS zu šarāpu „verbrennen“ vor, was allerdings neben der ge- 
bräuchlichen PuRS-Form šurpu sehr ungewöhnlich wäre; in diesem Falle könnte sum. sig/s $\mathrm{e}_{11}$ als unorthographische Schreibung für še $(\hat{\mathrm{g}})_{6}$ interpretiert werden.

ii 4 gišalam-ke-šè-re = salam riksi ,Statuette für eine Ritualzurüstung". Sum. ke-š̀̀-re ist offenbar eine syllabische Schreibung für kéš e $=$ riksu.

ii 5 'NíG ${ }^{\urcorner}-\mathrm{a} 1-$ šár-ra-ga = akalu zakûm „reine Speise“. Die - was das Sum. betrifft, vielleicht fehlerhafte - Gleichung erinnert an Hh XXIII 189 (MSL 11, 74): níg-àr-ra-sig 5 -ga = zakû.

ii 6-7 ti-ti $=$ mureššûm; te-te-h é $=$ mukiddu. Formen des Verbums ti-ti sind in OBGT III 232-239 (MSL 4, 74) paradigmatisch zusammengestellt (vgl. J. Black ${ }^{21991,15)}$ und teils durch rušš $\hat{u}$, teils durch den D-Stamm von kâdu wiedergegeben (in MSL mit "itch“ bzw. "tease“ übersetzt, zu ersterem vgl. CAD rašû $\mathrm{B}$, das $\mathrm{AHw}$. s.v. rašû II als „rot sein, werden" versteht). Viermaliges ti-ti (mit verschiedenen Bedeutungen) findet sich in Proto-Izi II 380-383 (MSL 13, 51). Der Gebrauch der beiden offenbar sinnverwandten Lemmata wird am Ende unserer Tafel durch ein zweigliedriges Sprichwort illustriert (v 5-8), worin ti-ti und te-te-h é bzw. ihre akk. Entsprechungen einen Parallelismus bilden.

Die ti-ti entsprechende akk. Form lautet dort mu-re-ši. Demnach handelt es sich um ein Partizip des D-Stamms von einer Wurzel tertiae infirmae. In Frage kommt vor allem das in AHw s.v. ruššû II und in CAD s.v. ruššû A verzeichnete, zu hebr./aram. $r$ - - $^{-}{ }^{c}$ „böse (sein)“ gehörige Verbum, das im Kontext des Sprichworts etwa „verunglimpfen“ heißt. Beide Wörterbücher rechnen die mit sum. te-te und ti-ti geglichenen Formen diesem Verbum zu.

Die te-te-hyé entsprechende Partizipialform lautet im Sprichwort $m u$-ki-id. Sie gehört zu einem Verbum mediae infirmae, und zwar wohl zu dem in CAD s.v. kâdu B verzeichneten Verbum, für das dort Parallelismen mit ašāšu und anāhu notiert sind und als Bedeutung fragend „to be distressed" angesetzt wird. Dasselbe Partizip liegt wohl auch in Silbenvokabular A 69 vor (H. de Genouillac 1928, 125 ii 15, s. Sollberger 1965, 26): mukiddum und mukabbirum sind dort mit $\mathrm{DU}_{8} \cdot \mathrm{DU}_{8}$ geglichen.

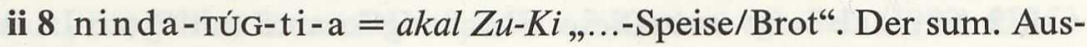
druck ist mir unklar, möglicherweise ist die Schreibung z.T. unorthographisch (etwa ti-a für dé-a, vgl. NINDA.ì.DÉ.A = mirsu?). Hinter mehrdeutigem $Z u$-Ki können sich $z \bar{u} k u$ „Reinheit“, sukku „Heiligtum“, sūqu „Straße" oder (m)usukku „Unreiner" verbergen.

ii $9 \mathrm{kaš-en-te-en} \mathrm{=} \mathrm{šikar} \mathrm{ši-Gi} \mathrm{,...-Bier“.} \mathrm{en-te-en} \mathrm{steht} \mathrm{hier} \mathrm{wohl}$ kaum in der geläufigen Bedeutung „Winter“. Die akk. Entsprechung gehört entweder zu šĭgu „Klage“ oder zu š̃qu „Tränken“. 
ii 10 ninda-ir-ĝar-ra $=a-k a-l u-m a ? e-z u$ - $u$. Der sum. Ausdruck ist mir ebenso wie seine akk. Entsprechung unklar. Letztere enthält akalu „Speise“ (oder akālu „essen“?). Falls das nächste Zeichen MA ist, würde es sich um die enklitische Partikel -ma (in der Bedeutung „ebenfalls“ oder zur Kennzeichnung eines adverbiellen Infinitivs?) handeln. Sollte das zweite Wort ezû „cacare" oder ein zu ir = zu'tu "Schweiß“ (Wurzel $w-\underline{d}-$ ) gehöriges Verbum/Verbaladjektiv sein?

ii 11-14 Die Gruppe weist Ähnlichkeiten mit dem Nippur-Vorläufer zu Hh XIII 64-69 (MSL 8/1, 84) auf (übereinstimmende Lemmata in Fettdruck):
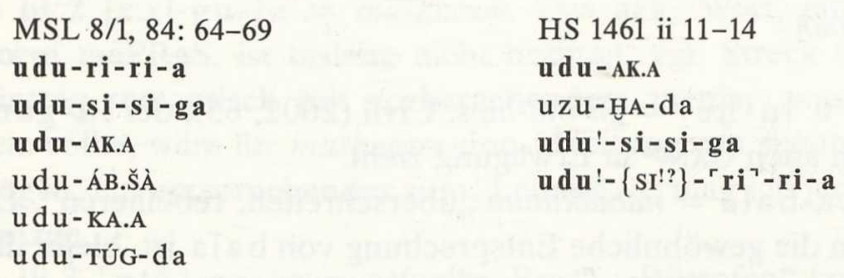

ii $11 \mathrm{udu}-\mathrm{AK} . \mathrm{A}=\check{s} a$ šapar-šu kīsu. Das sum. Lemma ist mit uduA.AK (oder u du-KA.A?) im Nippur-Vorläufer zu Hh XIII (Z. 66 bzw. 68) zu vergleichen. Die akk. Entsprechung dürfte „(Schaf,) dessen Schenkel gehäutet ist" bedeuten.

ii $12 \mathrm{uzu-HA-da} \mathrm{=} \mathrm{širum} Z a-a K-l u, \ldots$ Fleisch“. Das zu širum gehörige Verbaladjektiv gehört vielleicht zu sakālu „sich unrechtmäßig aneignen", doch sehe ich keine Verbindung mit sum. HA-da. In letzterem könnte man eine syllabische Schreibung für hád-da "getrocknet" vermuten, was aber nicht zu Za-aK-lu stimmt. Möglicherweise ist - was einen Fehler involvieren würde - udu-TúG-da im Nippur-Vorläufer zu $\mathrm{Hh}$ XIII (Z. 69) zu vergleichen.

ii $13 \mathrm{udu}$ !-si-si-ga $=k i-h u-d u-u m$. Das sum. Lemma dürfte mit udu-si-si-ga im Nippur-Vorläufer zu Hh XIII (Z. 65) zu vergleichen sein. Die akk. Entsprechung ist mir allerdings unklar.

ii $14 \mathrm{udu} !-\left\{\mathrm{sI}^{!}\right.$? $\}-\mathrm{ri}^{\urcorner}-\mathrm{ri}-\mathrm{a}=$ purrusum. Das zweite Zeichen ist als Dittographie von sI in der vorigen Zeile zu tilgen. Das sum. Lemma läßt sich dann mit u du-ri-ri-a im Nippur-Vorläufer zu Hh XIII (Z. 64) vergleichen. Die akk. Entsprechung gehört wohl als Infinitiv oder Verbaladjektiv zu parāsu D im Sinne von ,zergliedern“, vgl. die AHw. s.v. parāsu D 3) b) zitierte Stelle CT 17, 5: 53, wo sich ri-ri und purrusu entsprechen: šah-tur-ra á-úr-ne-ne [ù]-me-ni-ri-ri / MIN-a a-na mešre-ti-šú pur-ri-is-ma „zergliedere ein Ferkel“.

ii 15 úugu-GÁL = ka-sí-Bu. UGU und GÁL (falls so zu lesen) sind in Ligatur geschrieben. Aufgrund der akk. Entsprechung, die wohl mit dem in 
AHw. s.v. kusīpu, $k a(s) s i p p / b u$, in CAD s.v. kassibu und kusīpu B verzeichneten Pflanzennamen identisch ist, könnte man in „GÁL“ auch ein fehlerhaftes ZI oder zíB vermuten: *ugu-zi/zíb > kusīpu?

ii 16-17 še-er-gán = barīum "Schimmer“, še-er-zi = šarūrum „Glanz". Beide Gleichungen sind oft bezeugt (vgl. die Wörterbücher).

ii 18 lú-ḩi-a = gitmātūtum. Das akk. Wort ist entweder maskuliner Plural des Adjektivs gitmālum ,gleich“, „vollkommen“ oder das Abstraktum dazu. Zu vergleichen sind PRAK 2, P1.8, C.38: 5-7

hुé $\begin{aligned} & l u-\hat{u} \\ & \\ & \\ & l i-\hat{l} \vec{l} \\ & g i-i t-m a-[l u(m)]^{15}\end{aligned}$

und BM 23330 i 6: lú-ḩébi = git-ma-lu, s. Civil $(2002,65)$, der lú-gandùg liest, jedoch auch GANbe in Erwägung zieht.

ii 19 ŠUBUR.UR-bala = nabalkutum ,überschreiten, rebellieren“. Da nabalkutum allein die gewöhnliche Entsprechung von bala ist, bleibt der Sinn der beiden ersten Zeichen rätselhaft; möglicherweise ist ur als Lautindikator zu šubur aufzufassen.

ii 20 zú-ar $=k u-z a-z u-u m$. Das akk. Wort dürfte mit kuzāzum „Holz-

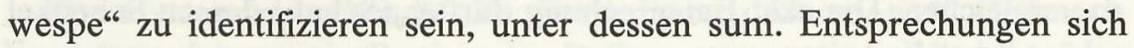

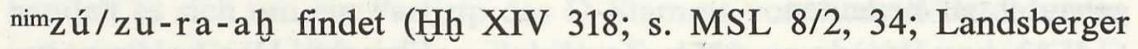
1934, 24f.). zú-ar wäre demnach eine Verballhornung von zú/zura-ah.

ii 21 e-lam-ma = mekk $\hat{u}$ „Treibstock“. Im Sum. liegt eine syllabische Schreibung für (gišs) $11 \mathrm{a} \hat{\mathrm{g}}$ (LAGAB) vor, die einen ursprünglichen $\hat{\mathrm{g}}$-Auslaut des Wortes reflektiert, wie er $\mathrm{m}$.W. bisher noch nicht bezeugt ist.

ii 22 UD = diqāru „Topf“. Sum. UD steht entweder graphisch fehlerhaft oder aber syllabisch ( $u t u$ ) für gewöhnliches KAM = útul.

ii 23 Lú!? $\times^{r} \mathrm{X}^{\top}$.RI = hanāqum, "erwürgen“. Das intendierte sum. Lemma ist vielleicht eri $i_{13}$ (LÚXÉŠ/ŠU)ri.

ii $24 \mathrm{KA} \times \mathrm{NE}-\mathrm{ZAR}$ ?.ZAR ${ }^{\text {? }}=e z \bar{e} z u$,zürnen“. Mit dem sum. Lemma ist Ea III 121 (MSL 14, 308; ergänzt nach Exzerpt CT 11, 28 iv 3', s. MSL $14,519)$ zu vergleichen: mùrgu $(\mathrm{KA} \times \mathrm{NE})=$ libbātu „Wut“.

ii 25 si-gi-ri = qá-ar-nu „Horn“. In dem sum. Ausdruck ist gewöhnliches $\mathrm{si}=$ qarnu durch unklares gi-ri erweitert. Vielleicht steht letzteres für ĝíri(-tab) „Skorpion“, dessen Greifer „Hörner" genannt wurden, vgl. Cavigneaux (1995, 80 mit Anm. 15); CAD s.v. qarnu 2.

15 In CAD s.v. gitmālu mit Lesung gan zitiert. 
ii $26 \mathrm{si-GI}=n \bar{a} t i$ qarni. Die beiden letzten Zeichen AR und NI sind partiell von der Rückseite her überschrieben (Ende von iii 2). Der akk. Ausdruck bedeutet „mit den Hörnern stoßend“, wobei wie zu erwarten $\mathrm{si}=$ qarnu. GI steht vielleicht mit dem Lautwert $\operatorname{sig}_{17}$ unorthographisch für sìg "schlagen“.

iii $1[\mathrm{x}] \cdot{ }^{\mathrm{x}} \mathrm{x} \cdot \mathrm{x} \cdot \mathrm{x}^{\urcorner}={ }^{r} m e-s u^{\top}-u m$. Für das akk. Wort kommen verschiedene Interpretationen in Betracht. In Hinblick auf das folgende Lemma kommt mesûm "waschen" in die engere Wahl, doch lassen sich die davorstehenden Zeichenreste nur schwer zu einem entsprechenden sum. Ausdruck, etwa $[\mathrm{a}]-{ }^{\top} \mathrm{tu}_{5}{ }^{7}$, ergänzen.

iii 2 [x.x]-gu-la = mašhatum. Das akk. Wort, ein Substantiv der Form maPRaS, ist bislang nicht bezeugt, vgl. Streck (2002). Falls der Eintrag semantisch mit vorhergehendem mesûm "waschen" assoziiert sein sollte, wäre für mašhatum eine Ableitung von šahătu „spülen“ zu erwägen, ein entsprechendes sum. Lemma vermag ich jedoch nicht zu ergänzen.

iii 3 'níĝ ’-sa-sa = qalqallu „Rost“, „Röstofen“ (zu qalû „rösten“). Die Gleichung ist auch in Hh X 384 bezeugt: MSL 7, 98 mit Ergänzung MSL 9, 193; Civil $(1996,152)$.

iv 1 [x-suk] ud = zuqquru ,erhöhen“ oder "sehr hoch“. Für das zweite Zeichen des akk. Wortes könnte man auch eine Lesung $\mathrm{MU}^{\text {! in }}$ Erwägung ziehen, was șmmuru „wünschen“ (š̀̀/téš sè-s è-ga; sá-sá; sậ-dúb) oder zummuru „besingen“ (š̀̀r; $d_{12}-d_{u_{12}}$ ) ergäbe. Der erhaltene Rest des sum. Lemmas paßt jedoch zu keiner der erwähnten Gleichungen, wohl aber zu SUKUD, weshalb ich die Lesung $z u-q u u^{\prime}-r[u]$ vorziehe.

iv $2 \mathrm{NI} . \mathrm{ZU}$ ? $\mathrm{HAR}=\mathrm{E}^{?}-\mathrm{Ku} \mathrm{r}^{\mathrm{r}} \mathrm{x}^{\mathrm{T}}\left[-\mathrm{x}^{?}\right]$. Unklar.

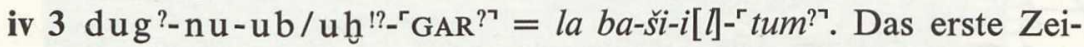
chen ist weder den Formen von BI (i 5; i $17 \mathrm{f}$., v 2) noch denen von GA (i 15, i 22, ii 22, ii $25 \mathrm{f}$., iii 3, v 7) eindeutig zuzuordnen. Die akk. Wiedergabe enthält keine direkte Entsprechung dieses Elements. Sie besteht aus einem mit $l \bar{a}(=$ sum. $\mathrm{nu})$ negierten, substantivierten femininen Verbaladjektiv bašiltu „nicht gar“, „unreif“ (die gewöhnliche sum. Entsprechung wäre š ${ }_{6}$ ).

v 1-4, „Weil ich eine Witwe bin, rieselt (es) täglich (aus) ... von ...-Mehl (wie Regen!?) auf mich" / „Weil ich eine Witwe bin, rieselt (es) täglich wie (aus) ein(em) Korb (?) von ...-Mehl auf mich". Offenbar ein Sprichwort wie v 5-8, im Unterschied zu jenem sind jedoch keine Beziehungen zum lexikalische Abschnitt erkenntlich.

Die Entsprechung zwischen sum. und akk. Fassung ist, was gú!?, A.AN!? bzw. $z a-{ }^{\ulcorner} b^{\prime} l^{\urcorner}$betrifft, problematisch. Das versuchsweise gú !? gele- 


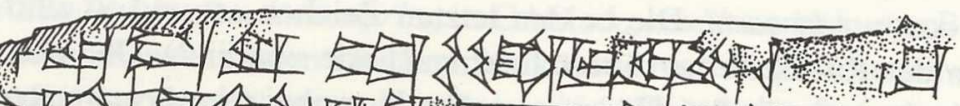

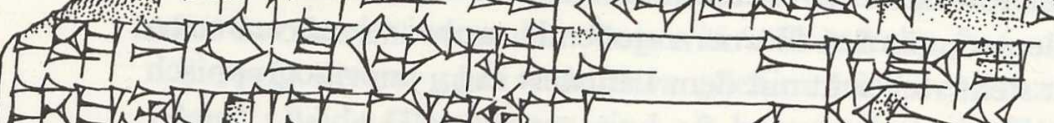

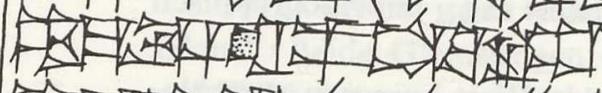

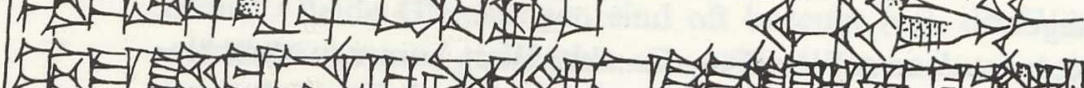

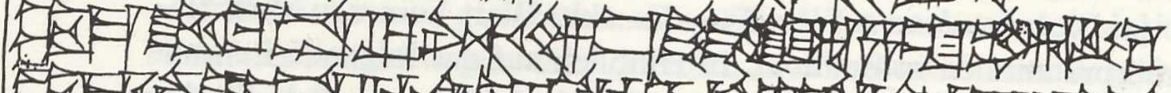

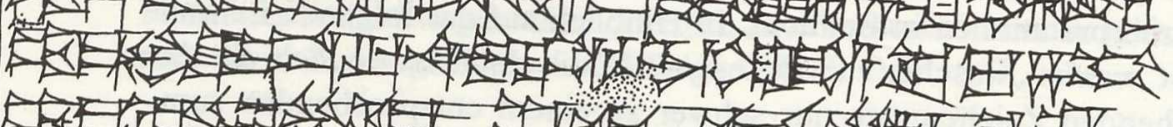
L

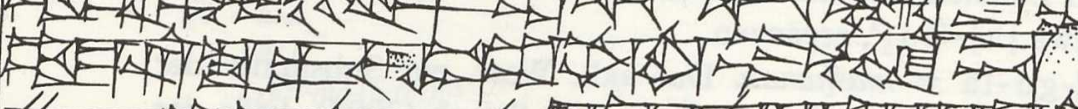

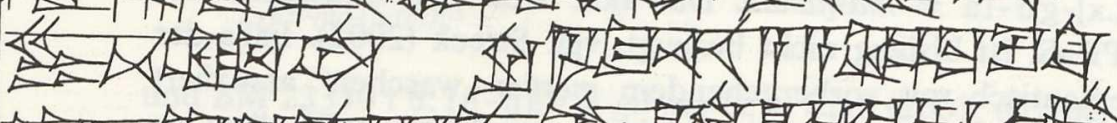
N1

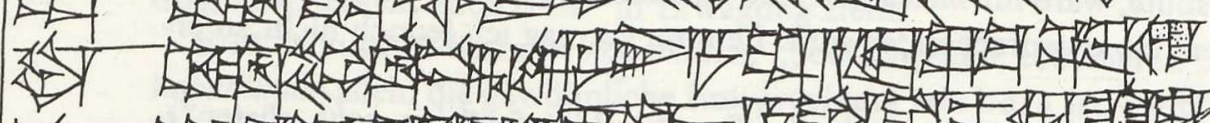

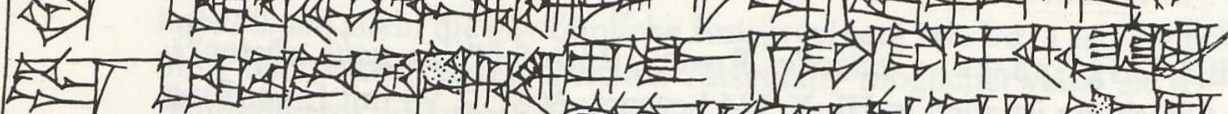

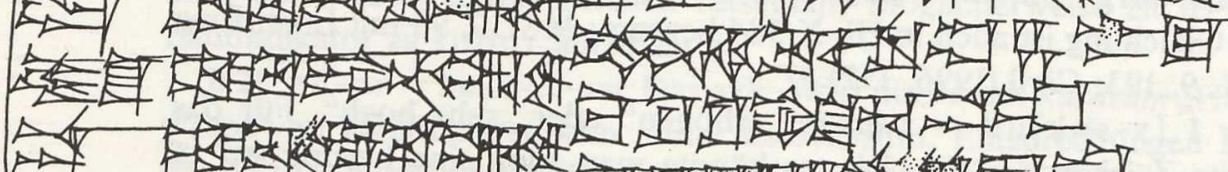

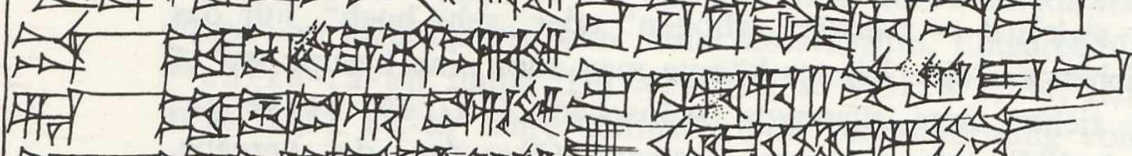

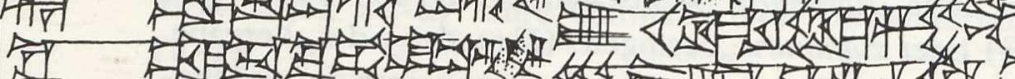
i. 7. A -15 年

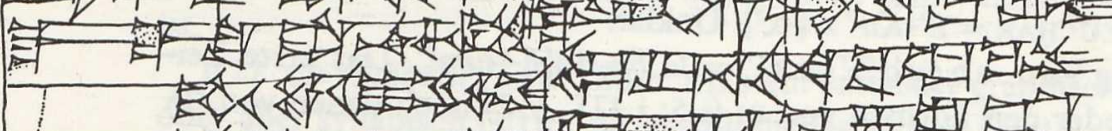
T r n 负

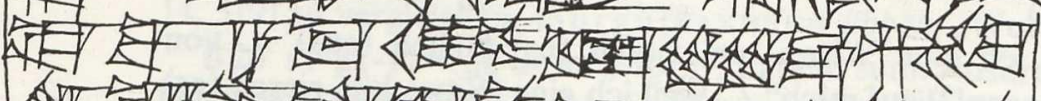
4I

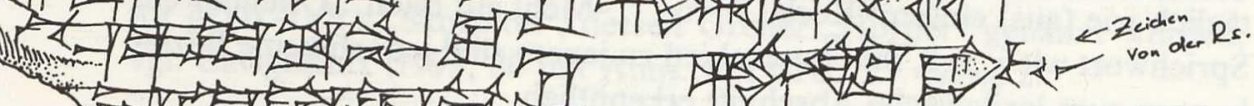
$\Rightarrow$ 政 
Wörter und Sprichwörter: der zweisprachige Schultext HS 1461

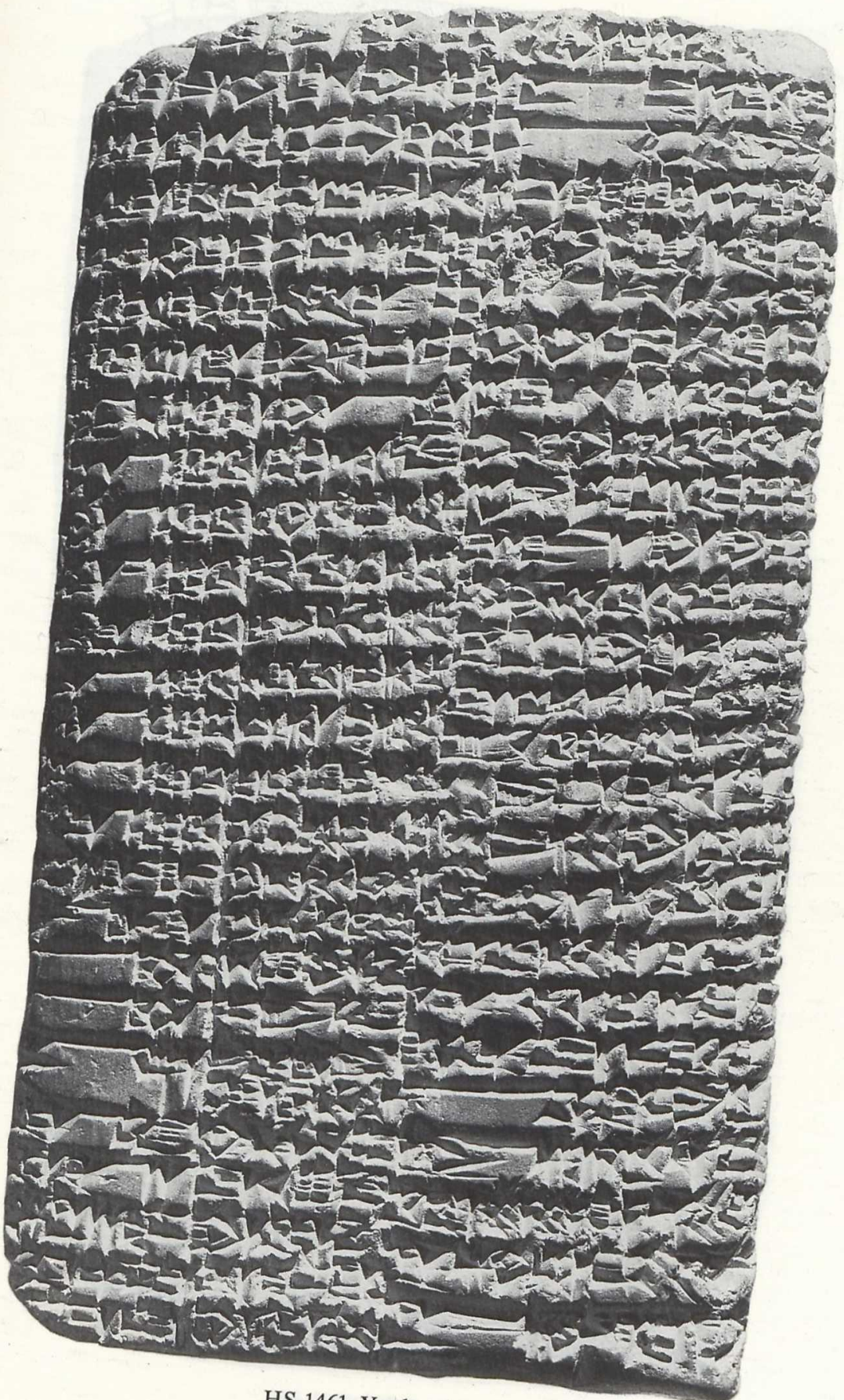

HS 1461, Vorderseite 


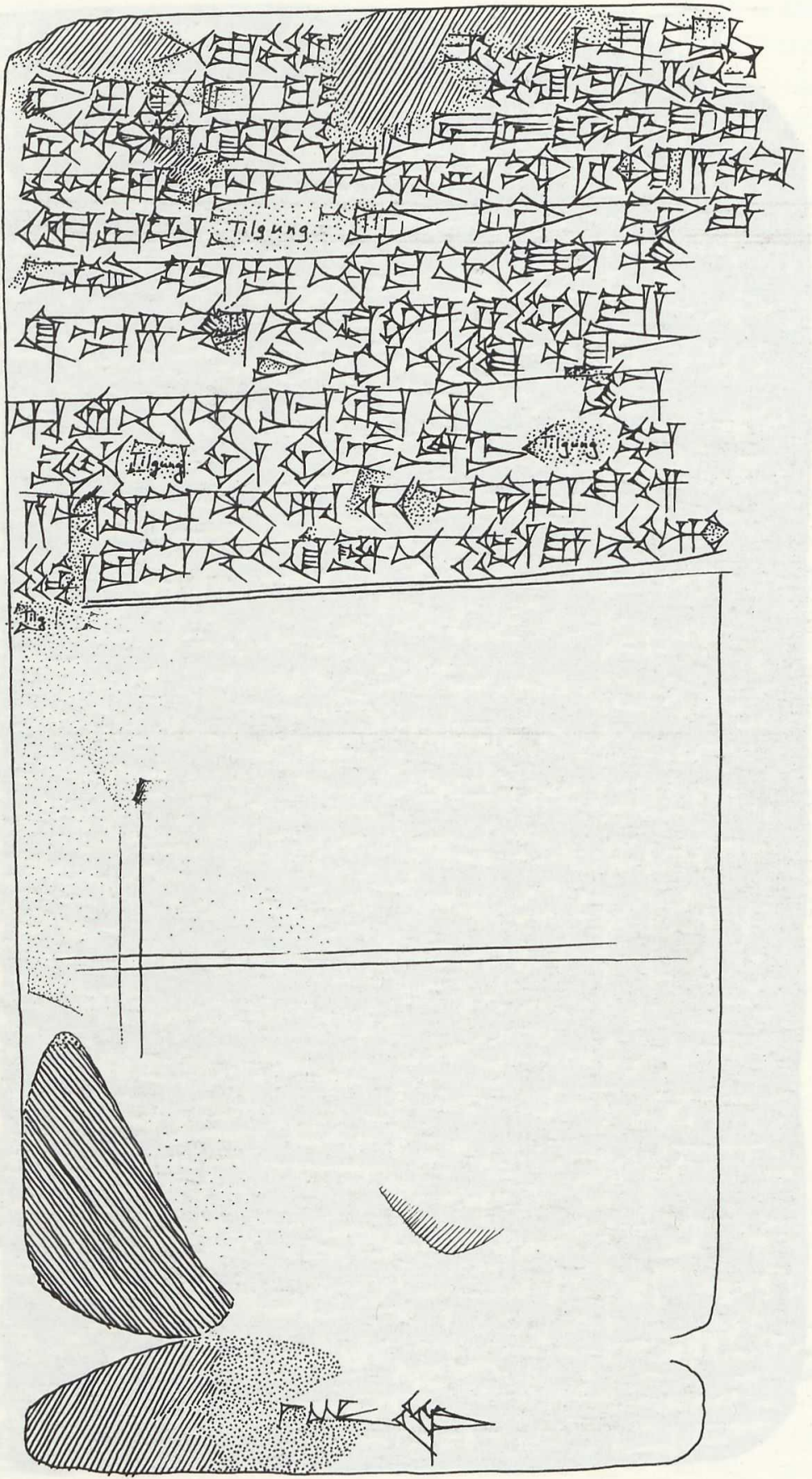

HS 1461, Rückseite 


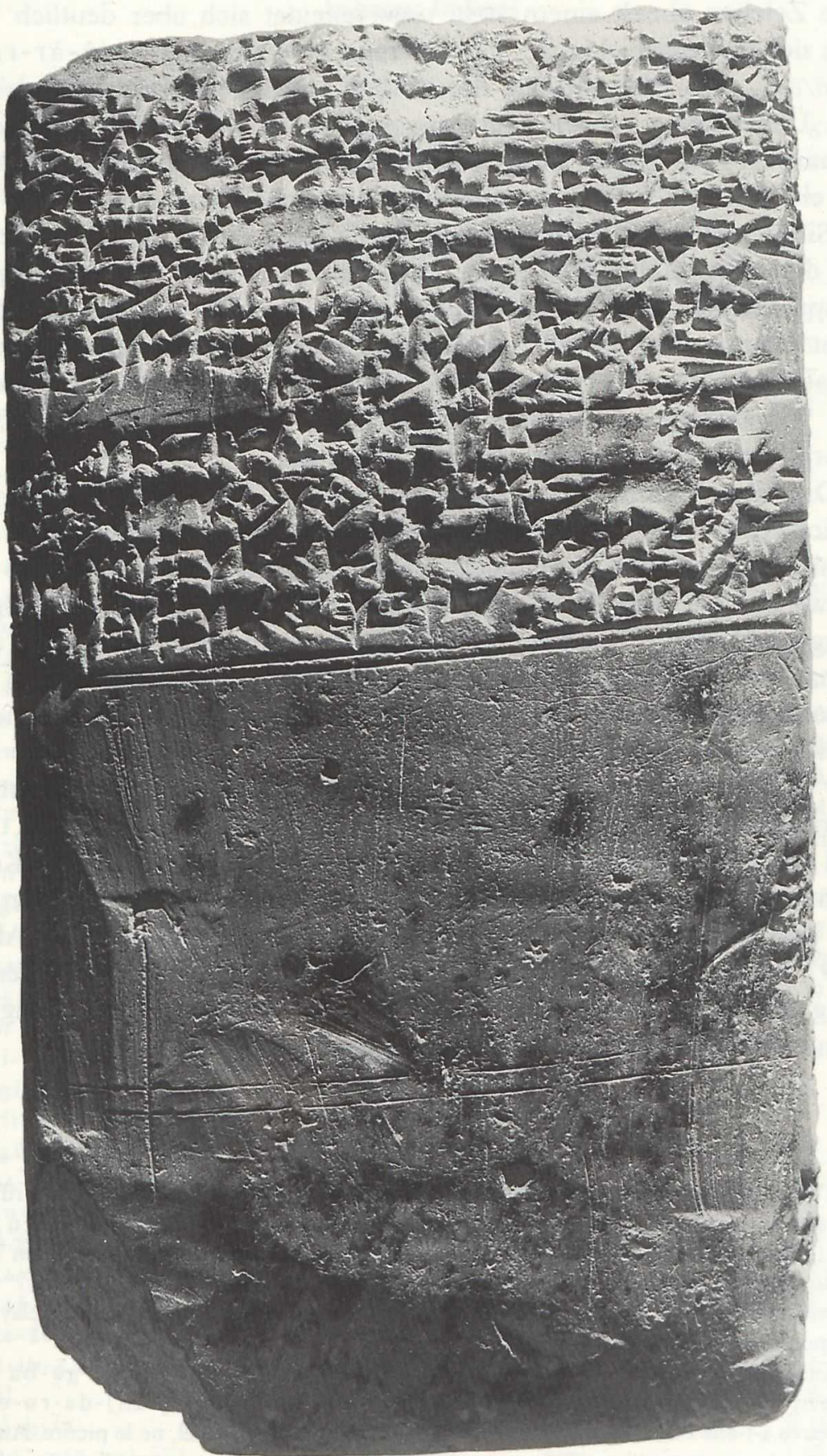

HS 1461, Rückseite 
sene Zeichen ähnelt einem šUM, unterscheidet sich aber deutlich von dem sicheren Beispiel in v 3. Die Lesung gú!? ergäbe gú!?-níg $-a ̀ r-r a=$ kiššă/ēnu „eine Hülsenfrucht, deren Mehl verbacken wird“ (AHw.). Die akk. Übersetzung scheint allerdings ní $\hat{\mathrm{g}}-\mathrm{à} \mathrm{r}-\mathrm{ra}=$ mundum / muddum vorauszusetzen. Damit bleiben gú!? und A.AN!? übrig, denen im Akk. nur ein Wort, $z a^{-}{ }^{-} b_{i} l^{?}$, gegenübersteht. Möglicherweise war A.AN!? = à m im Sinne von „wie“ intendiert und wurde dann durch - gin ${ }_{7}$ ersetzt, so daß die beiden Zeichen zu tilgen wären. Ein sum. A.AN!? = š̀ $\hat{g}$ "Regen“ entsprechendes Wort (zanānu, zunnu) ist in der akk. Fassung jedenfalls nicht zu erkennen; $z a-{ }^{r} b i l^{l^{\urcorner}}$scheidet hierfür trotz des vielversprechenden Anlauts aus, das Wort scheint eher die Entsprechung von gú ${ }^{\text {? }} \mathrm{zu}$ sein. Die vorgeschlagene Deutung ist allerdings problematisch, da zabbilu „Korb“ den Lexika zufolge erst spät belegt ist.

Dasselbe Verbum /biz/ kommt auch in dem spät überlieferten Sprichwort K $4347+16161$ iii 50-55 vor ${ }^{16}$. Ähnlichkeit weist ferner ein Passus in „Enki und Ninhursaĝa“ auf, wo davon die Rede ist, daß der Witwe das auf dem Dach ausgebreitete Malz nicht mehr von Vögeln weggepickt wird. ${ }^{17}$ Der Sinn des Sprichworts ist jedoch nicht leicht auszumachen. Möglicherweise liegt der Schlüssel zum Verständnis in der Assoziation von Mehl mit Totenopfern ${ }^{18}$ : die (von Almosen lebende?) Witwe wäre dann mit einer Toten verglichen.

v 5 „Der Narr ist ein Verunglimpfer der/des Helden, der Tor ist ein Störenfried der Mächtigen“. Das Sprichwort ist durch die Lemmata ti-ti und te-te-ḩé mit der lexikalischen Liste verknüpft, s.o. ii 6-7 mit Kommentar. Die erste der beiden formal und inhaltlich parallelen Zeilen findet sich (ohne akk. Übersetzung) in Sprichwortsammlung 3, s. Alster (1997, 98 Nr. 102): [h] u-ru-um ti-ti ur-sag -e-ne. Alsters Übersetzung „Fools are the lives of heroes“ wird durch unseren Text korrigiert, dem zufolge ti-ti und te-te-hé partizipial aufzufassen sind.

16 BWL 243 mit Kommentar S. 249, wo auf Falkenstein $(1952,127)$ verwiesen wird: é-a e sir-è-a; SIG $_{4}$.AL.ÙR.RA-ta; al-tu š-en he-pí; im-ma lag sa ĝ-ĝ́ ugu-ĝu ${ }_{10}$ al-

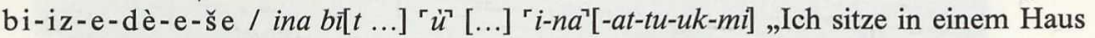
aus Asphalt und Backstein, sollte mir da ein Lehmklumpen auf den Kopf fallen?" (Falkenstein) bzw. „I live in a house of pitch and baked brick, (yet) a lump of clay falls upon my head“ (W.G. Lambert).

17 Attinger (1984) 8f., Z. 19-21: nu-mu-un-su/sú munu ù̀r-ra bàra-ga-ba mušen(-e) an-na munu 4 -bi na-an-gu $u_{7}$ e tu ${ }^{\text {mušen-e }}$ nu-mu(-un)-da-ru-e „La veuve a-t-elle étalé du malt sur le toit, aucun oiseau, dans le ciel, ne le picore. Aucune colombe ne se rengorge (?)“.

18 Vgl. Powell $(1986,14)$. 


\section{Anhang: Indices}

\section{Sumerisch(-Akkadisch)}

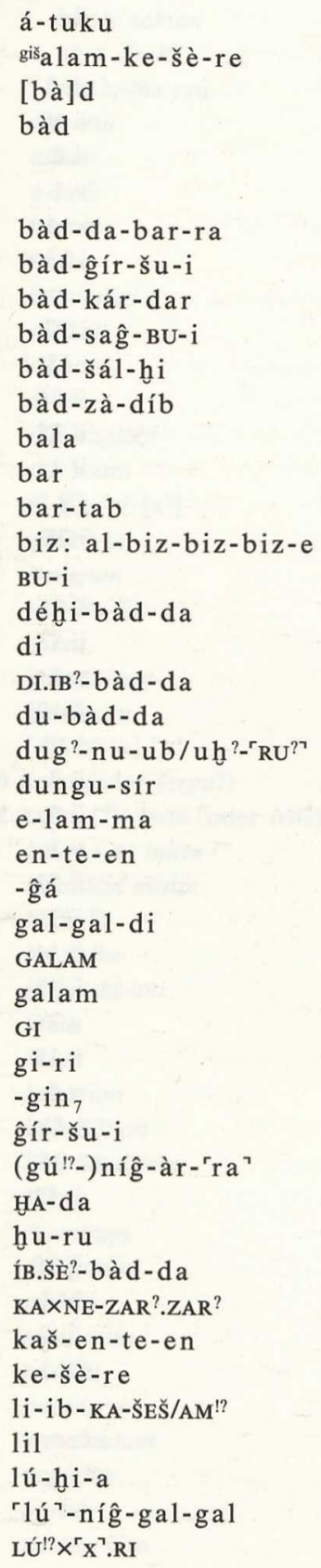

s. bàd-s a $\hat{g}-$ BU-i

tehûtum

s. gal-g al-di

pattabar dūrim

sēt dūrim

lā ba-ši-i $[l]-{ }^{r}$ tum $^{\text {? }} \quad$ iv 3

šapitum i 24

mekkû

s. kaš-en-te-en

s. ugu-ĝá

ša narbîm

irtum

simmiltu

s. si-GI

s. si-gi-ri

kìma

v 1

s. bàd-gír-šu-i

muddu

s. uzu-HA-da

ahurrûm

initti (oder irti?) dūrim

ezēzu

ii 24

šikar ši-Gi

s. giša la m-ke-š è-re

' hu/ri'-[x]-' $\mathbf{x}^{7}$

ii 1

lillum

gitmālūtum

ii 18

ša narbîm 


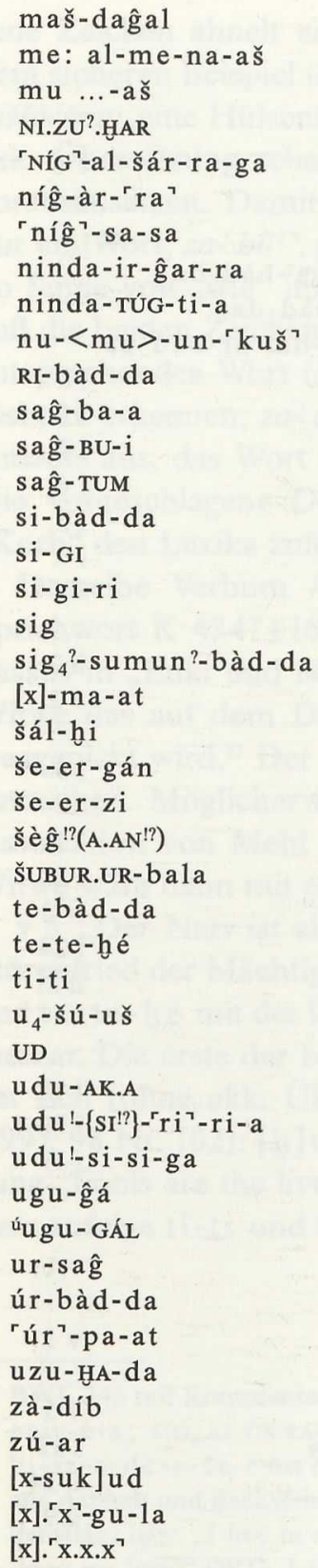

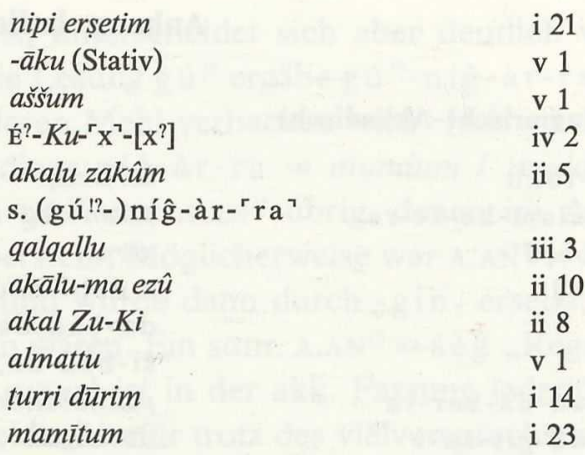

s. bà d-s a g - BU-i

qanānum

suqat dūrim

i 15

nāti qarni

ii 26

qarnu

ii 25

inbu (oder šerpu)

ii 3

tēru

šalmu

s. bàd-šá1-ḩi

barīrum

ii 16

šăurum

ii 17

v 1

nabalkutum ii 19

lēt dūrim i 10

$k a ̂ d u \mathrm{D}$

ii $7 ; \mathrm{v} 6$

rešû $\mathrm{D}$

ii 6 ; v 5

v 1

ii 22

ümišam

ii 11

ša šapar-šu kīṣu

ii 14

purrusum

ii 13

$k i-h u-d u-u m$

v 2

ii 15

$k a-s i-B u$

v 5

i 11

i 25

e/it-Bu-Zu

ii 12

sirum Za-aK-lu

ii 20

kuzāzum

iv 1

zaqāru D

iii 2

mašhatum

iii 1 


\section{Akkadisch(-Sumerisch)}

\begin{tabular}{|c|c|c|}
\hline \multirow{2}{*}{\multicolumn{3}{|c|}{$\begin{array}{l}\text { ahurrû } \\
\text { ak(a)lu: }\end{array}$}} \\
\hline & & \\
\hline akalu zakûm & 'NÍG’-al-šár-ra-ga & ii 5 \\
\hline akal Zu-Ki & ninda-TúG-ti-a & ii 8 \\
\hline akālu-ma ezû & ninda-ir-gar-ra & ii 10 \\
\hline almattu & nu-un- ${ }^{r}$ kuš & v 1 \\
\hline aššum & $\mathrm{mu} \ldots-\mathrm{aš}$ & v 1 \\
\hline$a-\check{s} u-\dot{u}$ & $\mathrm{~b} a \mathrm{r}-\mathrm{tab}$ & ii 2 \\
\hline barīru & še-er-gán & ii 16 \\
\hline bašlu & s. lā bašiltum & \\
\hline bēl emūqi & á-tuku & v 6 \\
\hline diqāru & UD & ii 22 \\
\hline dūrum & bàd & i 1 \\
\hline dūru & s. sētu, inittu, išdu, lētu & \\
\hline dūr naglabi & bàd-ĝír-šu-i & i 5 \\
\hline dūr nītim & bàd-zà-díb & i 4 \\
\hline$\hat{\mathrm{E}}^{?}-K u^{-} \mathrm{r}^{\top}-\left[\mathrm{x}^{?}\right]$ & NI.ZU?.HAR & iv 2 \\
\hline eli: eli-ja & ugu-ĝá & v 2 \\
\hline emūqum & s. bēl emūqi & \\
\hline$e / i t-B u-Z u$ & rúr ${ }^{\urcorner}-p a-a t$ & i 25 \\
\hline ezēzu & $\mathrm{KA} \times \mathrm{NE}-\mathrm{ZAR}^{?} \cdot \mathrm{ZAR}^{?}$ & ii 24 \\
\hline gitmālūtum & lú-ḩi-a & ii 18 \\
\hline hanāqum & LÚ!? $x^{r} X^{\top} \cdot R I$ & ii 23 \\
\hline${ }^{r} h u / r i^{\top}-[\mathrm{x}]-{ }^{r} \mathrm{x}^{\top}$ & $1 \mathrm{i}-\mathrm{ib}-\mathrm{KA}-\mathrm{S} E S \check{S} / \mathrm{AM}^{! ?}$ & ii 1 \\
\hline inbu (oder šerpu?) & sig & ii 3 \\
\hline inittu (?): initti (oder irti?) dūrim & і́в.šÈ?-bàd-da & i 12 \\
\hline irtum s. a. inittu & GALAM & i 20 \\
\hline išdu: išid dūrim & úr-bàd-da & i 11 \\
\hline$k \hat{a} d u \mathrm{D}$ & te-te-hé & ii $7 ; \mathrm{v} 6$ \\
\hline$k a-s i-B u$ & úugu-GÁL & ii 15 \\
\hline$k i-h u-d u-u m$ & udul-si-si-ga & ii 13 \\
\hline kīma & $-\operatorname{gin}_{7}$ & v 1 \\
\hline kīṣu & s. ša šapar-šu kīṣu & \\
\hline kuzāzum & zú-ar & ii 20 \\
\hline lā bašiltum & dug ${ }^{?}-$ nu-ub/uh ${ }^{?-{ }^{r}} \mathbf{R U}^{?\urcorner}$ & iv 3 \\
\hline lētu: lēt dūrim & te-bàd-da & i 10 \\
\hline lillum & lil & v 6 \\
\hline mamītum & $s a \hat{g}-b a-a$ & i 23 \\
\hline mašhatum & {$[x] \cdot x^{7}-g u-1 a$} & iii 2 \\
\hline mekkû & $e-1 a m-m a$ & ii 21 \\
\hline me-su-um & {$[x] \cdot x \cdot x \cdot x^{\top}$} & iii 1 \\
\hline muddu & $\left(\mathrm{gú}^{! ?-}\right)$ níg $-\mathrm{àr}-^{\ulcorner} \mathrm{ra}^{\top}$ & v 1 \\
\hline$m u-p i_{4}-u m$ & bàd-sấg-Bu-i & i 6 \\
\hline nabalkutum & ŠUBUR.UR-bala & ii 19 \\
\hline naglabu & s. dūr naglabi & \\
\hline narbû & s. ša narbîm & \\
\hline natāku Gtn & biz: al-biz-biz-biz-e & v 2 \\
\hline
\end{tabular}


națu: nāṭi qarni

nīpu: nipi erșetim

nìtu

parāsu D

pardum

pat(t)abarrum

pat(t)abar dūrim

qalqallu

qanānum

qarnu

qarnu

qarrādu

rešû $\mathrm{D}$

riksu

sētu: sēt dūrim

simmiltu

suqtu: suqat dūrim

șalmu: șalam riksi

ša narbîm

ša narbîm-ma

ša šapar-šu kīṣu

šalmu

šapitum

šapru

šă̄urum

šerpu (?)

$s i-G i$

šikaru: šikar ši-Gi

sīrum Za-aK-lu

šulhûm

tēru

tehûtum

turri dūrim

ümišam

zabbīlu (?)

Za-aK-lu

zaqāru D

$\mathrm{Zu}-\mathrm{Ki}$
si-GI

maš-da ĝ a l

ii 26

s. dūr nītim

udu !-\{sI!? $\}-\mathrm{ri}^{\urcorner}-\mathrm{ri}-\mathrm{a}$

bàd-kár-dar

bàd-da-bar-ra

DI.IB?-bàd-da

rníg ${ }^{\urcorner}-\mathrm{sa}-\mathrm{sa}$

sa ĝ-TUM

si-gi-ri

s. natû

ur-sa $\hat{g}$

ti-ti

s. șalmu

du-bàd-da

galam

si-bàd-da

gišalam-ke-éš-re

`1ú '-níg-gal-gal

gal-gal-di

udu-AK.A

[x]-ma-at

dungu-sír

s. ša šapar-šu kīṣu

še-er-zi

s. inbu

s. šikaru

kaš-en-te-en

u zu - HA-da

bàd-šál-ḩi

sig $_{4}{ }^{2}$-sumun ?-bàd-da

déhi i-bà $d-d a$

RI-bà d-da

$\mathrm{u}_{4}$-šú-u š

$\mathrm{X}(?)$

s. šrrum $Z a-a K-l u$

[x-suk] ud

s. akal $\mathrm{Zu}-\mathrm{Ki}$ ii 9

ii 12

i 2

i 8

i 9

i 14

v 1

v 4

i 4

ii 14

i 7

i 3

i 16

iii 3

i 22

ii 25

v 5

ii 6 ; v 5

i 13

i 19

i 15

ii 4

i 17

i 18

ii 11

i 26

i 24

ii 17

iv 1

\section{Bibliographie}

Alster, B. (1997): Proverbs of Ancient Sumer. Bethesda.

Attinger, P. (1984): Enki et Ninhursaĝa, ZA 74, 1-52.

Black, J. (21991): Sumerian Grammar in Babylonian Theory. Studia Pohl 12. Roma.

Cavigneaux, A. (1995): La parade du scorpion dans les formules magiques sumériennes, ASJ 17, 75-99. 
Civil, M. (1987): Feeding Dumuzi's Sheep: The Lexicon as a Source of Literary Inspiration, in: F. Rochberg-Halton (Hg.), Language, Literature, and History: Philological and Historical Studies Presented to Erica Reiner (New Haven) 37-56.

Civil, M. (1996): HAR-ra = hubullu: Tablet X dug = karpatu, in: Sallaberger (1996) 129-159.

Civil, M. (2002): The Forerunners of marû and hamțu in Old Babylonian, in: T. Abusch (Hg.), Riches Hidden in Secret Places. Ancient Near Eastern Studies in Memory of Th. Jacobsen (Winona Lake) 63-71.

Falkenstein, A. (1952): Das Potentialis- und Irrealissuffix -e-še des Sumerischen, IF 60, 113-130.

de Genouillac, H. (1928): Curieux syllabaire de l'époque de la dynastie de Babylone, RA 25, 123-132.

Gesche, P. (2001): Schulunterricht in Babylonien im ersten Jahrtausend v. Chr. AOAT 275. Münster.

Hallo, W.W. (1990): Proverbs Quoted in Epic, in: T. Abusch e. a. (Hg.), Lingering over Words. Studies in Ancient Near Eastern Literature in Honor of William L. Moran. HSS 37 (Atlanta), 203-217.

Krebernik, M. (1997): Zur Interpretation von ARET 5, 24-26, in: B. Pongratz-Leisten e.a. (Hg.), Ana šadî Labnāni lū allik. Beiträge zu altorientalischen und mittelmeerischen Kulturen. Festschrift für Wolfgang Röllig. AOAT 247 (Neukirchen) 185-192.

Krecher, J. (1992): UD.GAL.NUN versus ,Normal' Sumerian: Two Literatures or One?, in: P. Fronzaroli (Hg.), Literature and Literary Language at Ebla. Quaderni di Semitistica 18 (Firenze) 285-303.

Landsberger, B. (1934): Die Fauna des Alten Mesopotamien nach der 14. Tafel der Serie HAR-RA = HUBULLU. ASAW 42/VI. Leipzig.

Michalowski, P. (1998): Literature as a Source of Lexical Inspiration, in: J. Braun e. a. (Hg.), Written on Clay and Stone. Ancient Near Eastern Studies Presented to Krystyna Szarzynska on the Occasion of Her 80th Birthday (Warsaw) 65-73.

Powell, M. (1986): mun-du as an Akkadian Loan Word in Sumerian, ZA 76, 12-16.

Robson, E. (2001): The Tablet House: A Scribal School at Old Babylonian Nippur, RA 95, 39-66.

Römer, W.H. Ph. (1988): Sumerische Hymnen (II), BiOr. 45, 24-60.

Sallaberger,W. (1996): Der babylonische Töpfer und seine Gefäße. MHE Mémoirs III. Ghent.

Selz, G. (1993): Altsumerische Wirtschaftsurkunden aus amerikanischen Sammlungen. FAOS $15 / 2$. Stuttgart.

Sjöberg, Å. (1996): UET 7 no.93. A Lexical Text or a Commentary?, ZA 86, 220-237.

Sollberger, E. (1965): A Three-Column Silbenvokabular A, in: H. G. Güterbock/Th. Jacobsen (Hg.), Fs. Landsberger. AS 16 (Chicago) 21-28.

Streck, M.P. (2002): Die Nominalformen maPRaS(t), maPRāS und maPRiS(t) im Akkadischen, in: N. Nebes (Hg.), Neue Beiträge zur Semitistik. Jenaer Beiträge zumVorderen Orient 5 (Wiesbaden) 223-257.

Veldhuis, N. (1997): Elementary Education at Nippur. The Lists of Trees and Wooden Objects. Diss. Groningen 1997. 